\title{
MicroRNA-146b inhibition augments hypoxia-induced cardiomyocyte apoptosis
}

\author{
JING-WEI LI, SI-YI HE, ZE-ZHOU FENG, LIANG ZHAO, WEI-KUN JIA, \\ PENG LIU, YUN ZHU, ZHAO JIAN and YING-BIN XIAO \\ Department of Cardiovascular Surgery, PLA, Xinqiao Hospital, \\ Third Military Medical University, Chongqing 400037, P.R. China
}

Received January 3, 2015; Accepted August 24, 2015

DOI: $10.3892 / \mathrm{mmr} .2015 .4333$

\begin{abstract}
MicroRNAs (miRs) regulate a number of physiological and pathological processes, including myocardial chronic hypoxia. Previous studies revealed that the expression of miR-146b is increased in vitro and in vivo following the induction of hypoxia. In the present study, the role of miR-146b in hypoxic cardiomyocytes, and the mechanisms underlying its activity, were investigated. The expression of miR-146b was measured in tissue samples from patients with congenital heart disease by reverse transcription-quantitative polymerase chain reaction. The rat $\mathrm{H} 9 \mathrm{c} 2$ cardiomyocyte cell line was transfected with an miR-146b inhibitor or the experimental controls, and the cells were maintained under hypoxic conditions for $72 \mathrm{~h}$. The expression of miR-146b increased following the induction of hypoxia. Transfection with the miR-146b inhibitor enhanced the release of lactate dehydrogenase and increased hypoxia-induced apoptosis, as determined by terminal deoxynucleotidyl transferase dUTP nick-end labeling, Hoechst 33258 staining, JC-1 assay (measuring mitochondrial membrane permeability) and annexin $\mathrm{V} /$ propidium iodide analysis. A decreased expression of Bcl-2 was observed, whereas the expression levels of cleaved-caspase 3 and Bax were increased. Western blot analysis and a dual luciferase reporter assay confirmed that ribonuclease $\mathrm{L}$ is a direct target of miR-146b. Furthermore, inhibition of miR-146b increased the activation of nuclear factor- $\kappa \mathrm{B}$ and signal transducer and activator of transcription 3 . In conclusion, the inhibition of miR-146b may increase hypoxia-induced cardiomyocyte apoptosis.
\end{abstract}

\section{Introduction}

Chronic hypoxia is one of the basic pathophysiological processes associated with congenital heart disease (CHD) (1).

Correspondence to: Dr Ying-Bin Xiao, Department of Cardiovascular Surgery, PLA, Xinqiao Hospital, Third Military Medical University, 183 Xinqiao Street, Chongqing 400037, P.R. China

E-mail: xiaoyb@vip.sina.com

Key words: microRNA-146b, RNase L, hypoxia, apoptosis
Although patients with cyanotic CHD suffer long-term chronic hypoxia, the majority are able to tolerate hypoxia until surgery. At present, the underlying mechanisms of CHD remain to be fully elucidated (2). MicroRNAs (miRNAs) are endogenous, small ( 16-29 nucleotides) non-coding, single-stranded transcripts, which negatively regulate gene expression post-transcriptionally by targeting the 3'-untranslated region (UTR) of mRNAs (3). miRNAs have been demonstrated to exert critical roles in a number of cardiac diseases, including cardiac hypertrophy, heart failure, arrhythmia and ischemia-reperfusion (I/R) injury (4). A previous report from our laboratory demonstrated that miRNAs are involved in the process of cardiomyocyte adaptation to chronic hypoxia (5). A previous study revealed that the expression of miR-146b was higher in the heart tissue of patients with the CHD defect, Tetralogy of Fallot (TOF), compared with that of normal infants (6). Furthermore, the expression of miR-146b also increased in human pulmonary arterial smooth muscle cells following the onset of hypoxia (7), and the levels of miR-146b were increased in the lung tissue in an experimental animal model of CHD compared with that of the control groups (8). Therefore, it was hypothesized that miR-146b may function as a regulator involved in the cardiac chronic hypoxia process. miR-146b is the key miRNA regulating the immune system, particularly the natural immunity pathway (9). This miRNA predominantly regulates the nuclear factor- $\kappa \mathrm{B}(\mathrm{NF}-\kappa \mathrm{B})$ pathway by directly targeting interleukin-1 receptor-associated kinase 1 (IRAK1), tumor necrosis factor receptor-associated factor 6 (TRAF6) and myeloid differentiation primary response gene 88 (MyD88) (10). However, the role of miR-146b in cardiomyocytes under hypoxic conditions remains to be fully elucidated. The present study aimed to investigate the changes in the expression levels of miR-146b in cardiomyocytes under hypoxic conditions, and to assess whether miR-146b has a protective role in myocardial adaptation to chronic hypoxia.

\section{Materials and methods}

Sampling of the myocardial section. The sampling procedure was performed, as previously described (5). Briefly, 21 infant patients with CHD, with an average age of 17.1 months, were eligible for enrollment in the present study. The inclusion criteria were: (i) infants were aged between 3.2 and 
60.0 months; (ii) a diagnosis of TOF had been made; (iii) symptoms of a ventricular septal defect (VSD) combined with either pulmonary atresia or a right ventricular outflow tract obstruction were present, which required surgical reconstruction. The diagnosis was obtained using echocardiography, and was confirmed at the time of surgery. The exclusion criteria implemented in the present study were infectious diseases, and other types of congenital malformation. A total of nine patients with TOF, and one with pulmonary atresia and a VSD, were enrolled in the cyanotic group, whereas 11 patients with a right ventricular outflow tract obstruction and a VSD were enrolled in the acyanotic group. All subjects were recruited from Xinqiao Hospital (Chongqing, China). The local ethics committee of the Third Military Medical University Affiliated Hospital approved the design of the present study, and this investigation conformed to the principles of the Declaration of Helsinki. Written informed consent was obtained from the parents of all patients. Routine procedures for anesthesia and surgery were performed, as previously described (1). The biopsies were obtained from the right ventricular outflow tract and were stored at $-80^{\circ} \mathrm{C}$ prior to analyses.

Cell culture. The heart-derived H9c2 cell line was obtained from American Type Culture Collection (Manassas, VA, USA) and was maintained in Dulbecco's modified Eagle's medium (DMEM; Invitrogen Life Technologies, Carlsbad, CA, USA), supplemented with $10 \%$ fetal bovine serum (Invitrogen Life Technologies) at $37^{\circ} \mathrm{C}$. Following incubation in serum-free medium overnight, the medium was changed to serum-contained DMEM and the cells were placed in a modular incubator (3131; Forma Scientific, Marietta, $\mathrm{OH}$, USA), containing a gaseous mixture of $94 \% \mathrm{~N}_{2}, 5 \% \mathrm{CO}_{2}$ and $1 \% \mathrm{O}_{2}$ at $37^{\circ} \mathrm{C}$ for 24,48 or $72 \mathrm{~h}$. The cells under conditions of normoxia $\left(21 \% \mathrm{O}_{2}\right)$ were classified as the control group.

Reverse transcription-quantitative polymerase chain reaction $(R T-q P C R)$. The total RNA was isolated using RNAiso Plus reagent (Takara Bio, Inc., Otsu, Japan). The cDNA library was created using a PrimeScript RT reagent kit (Takara Bio, Inc.). The RT-qPCR analysis was performed using the $\mathrm{SYBR}^{\circledR}$ Premix Ex Taq ${ }^{\mathrm{TM}}$ kit (Takara Bio, Inc,) on a ViiA 7 Real-Time PCR system (Applied Biosystems, Los Angeles, CA, USA). The specific stem-loop primers of miR-146b were obtained from Guangzhou RiboBio Co., Ltd. (Guangzhou, China). RT was performed at $42^{\circ} \mathrm{C}$ for $30 \mathrm{~min}$ and $85^{\circ} \mathrm{C}$ for $5 \mathrm{sec}$. The PCR protocol for miR-146b consisted of 1 cycle of $95^{\circ} \mathrm{C}$ for $30 \mathrm{sec}$, 40 cycles of $95^{\circ} \mathrm{C}$ for $5 \mathrm{sec}$ and $60^{\circ} \mathrm{C}$ for $34 \mathrm{sec}$. All reactions were repeated in triplicate and the quantitative expression data were normalized against U6 RNA.

miRNA transfection. Upon reaching 30-50\% confluence, the cells were transfected with $100 \mathrm{nM}$ miR-146b inhibitor (GenePharm, Shanghai, China) or the negative controls (which are random sequence miRNA inhibitor that has been widely tested in rat cell lines and shown not to affect miRNA function), using Lipofectamine $2000^{\mathrm{TM}}$ reagent (Invitrogen Life Technologies), according to the manufacturer's instructions. All transfections were performed $24 \mathrm{~h}$ following seeding, and the cells were incubated for a further $48 \mathrm{~h}$ prior to the hypoxia experiments.
Lactate dehydrogenase ( $L D H)$ assay. The membrane integrity of the cultured H9c2 cardiomyocytes was assessed by measuring the release of LDH into the culture medium using an LDH assay kit (Beyotime Institute of Biotechnology, Beijing, China). The results obtained were divided by the maximum release of LDH in each group in order to calculate the cell mortality rate. Briefly, the assay was performed in 96-well plates, and the absorbance was measured using a microplate reader (Varioskan ${ }^{\circledR}$ Flash; Thermo Fisher Scientific, Waltham, MA, USA) at $490 \mathrm{~nm}$.

Flow cytometric analysis. Following treatment, the cells were digested with trypsin, washed once with cold phosphate-buffered saline (PBS),then resuspended in $100 \mu 1 \mathrm{1X}$ Binding Buffer at a concentration of $1 \times 10^{5}$ cells $/ \mathrm{ml}$. They were subsequently incubated with $5 \mu \mathrm{l}$ annexin V-fluorescein isothiocyanate and $5 \mu \mathrm{l}$ propidium iodide (Annexin V:FITC Apoptosis Detection kit I; cat. no. \#556547; BD Biosciences, San Diego, CA, USA) for $20 \mathrm{~min}$ at room temperature for each tube. Then $400 \mu \mathrm{l}$ of $1 \mathrm{X}$ Binding Buffer were added to each tube. The cell suspensions were analyzed by flow cytometry (BD LSRFortessa X-20, BD Biosciences). The results were analyzed using FlowJo 7.61 software (Treestar Inc., Ashland, OR, USA).

Terminal deoxynucleotidyl transferase dUTP nick-end labeling (TUNEL). The extent of apoptosis was also analyzed by TUNEL staining using the Cell Death Detection kit (Beyotime Institute of Biotechnology), according to manufacturer's instructions. The slides were visualized under a DMI3000 fluorescence microscope (Leica, Mannheim, Germany). The total cell number in a selected area was determined on the basis of 4',6-diamidino-2-phenylindole (DAPI) nuclear staining. The apoptotic index was calculated as the number of TUNEL-positive, DAPI-stained cells divided by the total number of cells in five random fields.

Hoechst 33258 staining. The $\mathrm{H} 9 \mathrm{C} 2$ cells were detected using Hoechst 33258 staining (Beyotime Institute of Biotechnology), according to the manufacturer's instructions. The cells were washed with PBS and treated with Hoechst $33258(10 \mu \mathrm{g} / \mathrm{ml})$ for $50 \mathrm{~min}$ at $37^{\circ} \mathrm{C}$ in the dark. The Hoechst-stained nuclei were subsequently observed under a fluorescence microscope (Leica) at a wavelength of $521 \mathrm{~nm}$.

JC-1 staining. The apoptotic cells were assessed using the JC-1 assay kit (KeyGen Biotech Co., Ltd., Nanjing, China), according to the manufacturer's instructions. The JC-1 fluorescent probe was used to detect alterations in the mitochondrial transmembrane potential $\left(\Delta \Psi_{\mathrm{m}}\right)$, which is one of the earliest indicators of apoptosis occurring in cells. Following treatment, the cells were washed with PBS twice and incubated with the JC-1 stain in the dark at $37^{\circ} \mathrm{C}$ for $30 \mathrm{~min}$. The cells were subsequently observed under a fluorescence microscope (Leica).

Western blotting. The proteins were extracted from the H9C2 cells using sodium dodecyl sulfate (SDS) lysis buffer (Beyotime Institute of Biotechnology), containing $500 \mathrm{mM}$ phenylmethylsulfonyl fluoride, for $30 \mathrm{~min}$. A total 

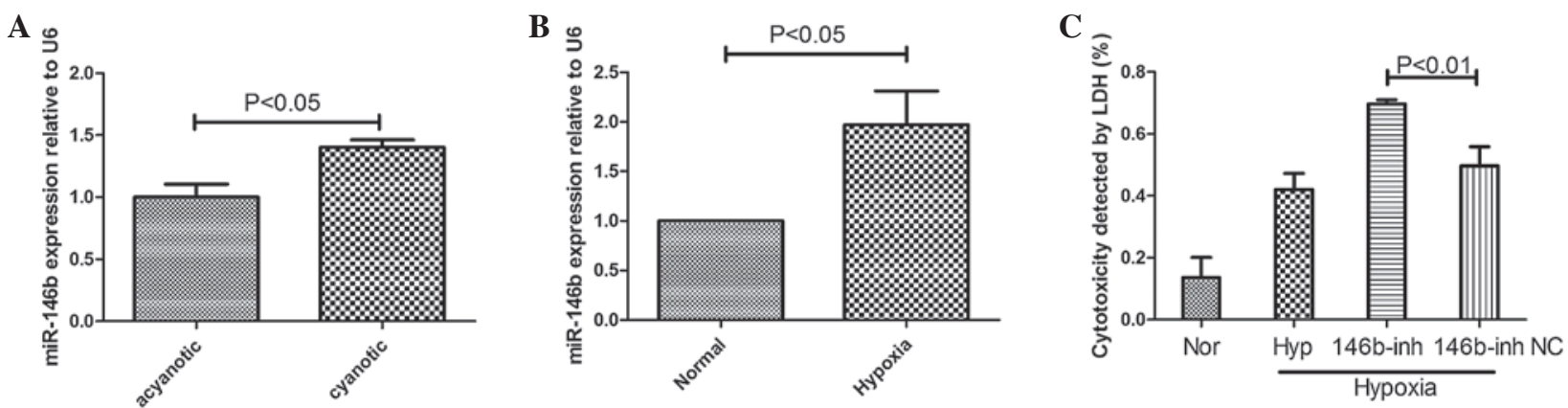

Figure 1. Expression of miR-146b in heart samples from patients with CHD and hypoxic cardiomyocytes. (A) Reverse transcription-polymerase chain reaction revealed that the expression of miR-146b was significantly upregulated in the heart tissue of patients with cyanotic CHD compared with acyanotic CHD $(\mathrm{P}<0.05)$. (B) The $\mathrm{H} 9 \mathrm{c} 2$ cells were cultured in $1 \% \mathrm{O}_{2}$ for $72 \mathrm{~h}$ and the expression of miR-146b increased when the cells were exposed to hypoxic conditions $(\mathrm{P}<0.05)$. (C) Cell mortality was determined by measuring the release of LDH under normoxic or hypoxic conditions after $72 \mathrm{~h}$, and with or without addition of a 146b-inh. The data are expressed as the mean \pm standard deviation $(\mathrm{P}<0.01,146 \mathrm{~b}$-inhibitor-treated cells compared with the NC). NC, normal control; Nor, normal; Hyp, hypoxia; inh, inhibitor; LDH, lactate dehydrogenase; miR, microRNA; 146b-inh, inhibitor of miR-146b; CHD, congenital heart disease.

of $40 \mu \mathrm{g}$ protein was separated by SDS-PAGE (Bio-Rad Laboratories, Inc., Hercules, CA, USA) and transferred onto a polyvinylidene difluoride membrane (Roche Diagnostics, Indianapolis, IN, USA). The concentration of separation gel was $12 \%$ for Bcl-2, Bax and cleaved-caspase 3 and $10 \%$ for the other proteins. The membranes were blocked with 5\% BSA for $1 \mathrm{~h}$ and incubated with primary antibodies at $4^{\circ} \mathrm{C}$ overnight, followed by incubation at room temperature for $1 \mathrm{~h}$ with secondary antibodies conjugated to horseradish peroxidase. The antibodies against $\mathrm{Bcl}-2$ (rabbit anti-rat; cat. no. \#2870; 1:1,000), Bax (rabbit anti-rat; cat. no. \#2772; 1:1,000), caspase 3 (rabbit anti-rat; cat. no. \#9662; 1:1,000), signal transducer and activator of transcription 3 (STAT3; (rabbit anti-rat; cat. no. \#9132, 1:1,000), phosphorylated (p-)-STAT3, p65 (rabbit anti-rat, cat. \#8242, 1:1000) and p-p65 (rabbit anti-rat; cat. no. \#3037; 1:1,000) were purchased from Cell Signaling Technology, Inc. (Danvers, MA, USA); the antibody against ribonuclease (RNase) L was obtained from Santa Cruz Biotechnology, Inc. (Santa Cruz, CA, USA; rabbit anti-rat; sc-22871; 1:700). The resultant bands were visualized using BeyoECL Plus kit (Beyotime Institute of Biotechnology).

Luciferase reporter assay. The miR-146b promoter, or fragments generated by mutagenesis (Guangzhou RiboBio Co., Ltd., Guangzhou, China), were cloned into the XhoI and NotI sites downstream of the luciferase reporter gene of pmiR-RB-Report (Guangzhou RiboBio Co., Ltd.). This plasmid contained Renilla luciferase as the reporter and firefly luciferase as the internal control. The plasmid DNA and $\mathrm{miR}-146 \mathrm{~b} \mathrm{mimic} / \mathrm{mimic}$-normal control (NC) were co-transfected into HEK293T cells using Lipofectamine ${ }^{\mathrm{TM}} 2000$ reagent (Invitrogen Life Technologies). The activity of luciferase was measured using the Dual-Glo luciferase assay system (Promega Corporation, Madison, WI, USA) at $48 \mathrm{~h}$ post-transfection.

Statistical analysis. The data were analyzed using GraphPad Prism 5.0 software (GraphPad Prism Inc., La Jolla, CA, USA) and are expressed as the mean \pm standard deviation. Comparisons between groups were made using one-way analysis of variance or two-tailed t-test. All experiments were repeated three times. $\mathrm{P}<0.05$ was considered to indicate a statistically significant difference.

\section{Results}

Expression of miR-146b is upregulated in samples from patients with cyanotic CHD. A previous study reported that miR-146b is expressed at higher levels in tissue samples of patients with TOF compared with the normal controls (6). The present study hypothesized that this was caused, at least in part, by hypoxia. The clinical characteristics of the patients enrolled in the present study were previously reported (5). A significantly lower $\mathrm{O}_{2}$ saturation in the arterial blood was observed in the cyanotic group (74.3\%) compared with the acyanotic group $(98.0 \%$; $\mathrm{P}<0.05)$. The levels of hemoglobin $\mathrm{Hb}$ and hematocrit (Hct) in the arterial blood were higher in the cyanotic group compared with the acyanotic group $(\mathrm{Hb}$, 171 , vs. $123 \mathrm{~g} / 1$; Hct, 54.1 , vs. $40.3 \%$; $\mathrm{P}<0.05$ ). Other factors, including gender, age and body weight, were similar between the groups. The present study revealed that the expression levels of miR-146b were $40 \%$ higher in cyanotic hearts compared with acyanotic hearts $(\mathrm{P}<0.05$; Fig. 1A).

Expression of miR-146b increases in cardiomyocytes under hypoxic conditions. The $\mathrm{H} 9 \mathrm{c} 2$ cells were cultured in $1 \% \mathrm{O}_{2}$ for $72 \mathrm{~h}$. The expression of miR-146b was assessed by RT-qPCR. The expression of miR-146b was significantly upregulated 0.97 -fold compared with that of the normal group $(\mathrm{P}<0.05)$ at 72 h (Fig. 1B).

Effect of miR-146b on cell apoptosis under hypoxic conditions. The induction of hypoxia increased the levels of LDH in the culture medium, and the downregulation of miR-146b resulted in a further increase of LDH (Fig. 1C). In order to confirm whether miR-146b was involved in hypoxia-induced apoptosis, several methods were used. Flow cytometric analysis revealed a higher level of cell apoptosis at $72 \mathrm{~h}$ following the induction of hypoxia, and transfection with the miR-146b inhibitor increased the apoptosis rate (17.3, vs. $7.3 \%$ under hypoxic conditions; $\mathrm{P}<0.05$; Fig. $2 \mathrm{~A}$ ). To further assess the effect of miR-146b, TUNEL was performed. As shown in Fig. 2B, the apoptotic index was significantly increased 
A
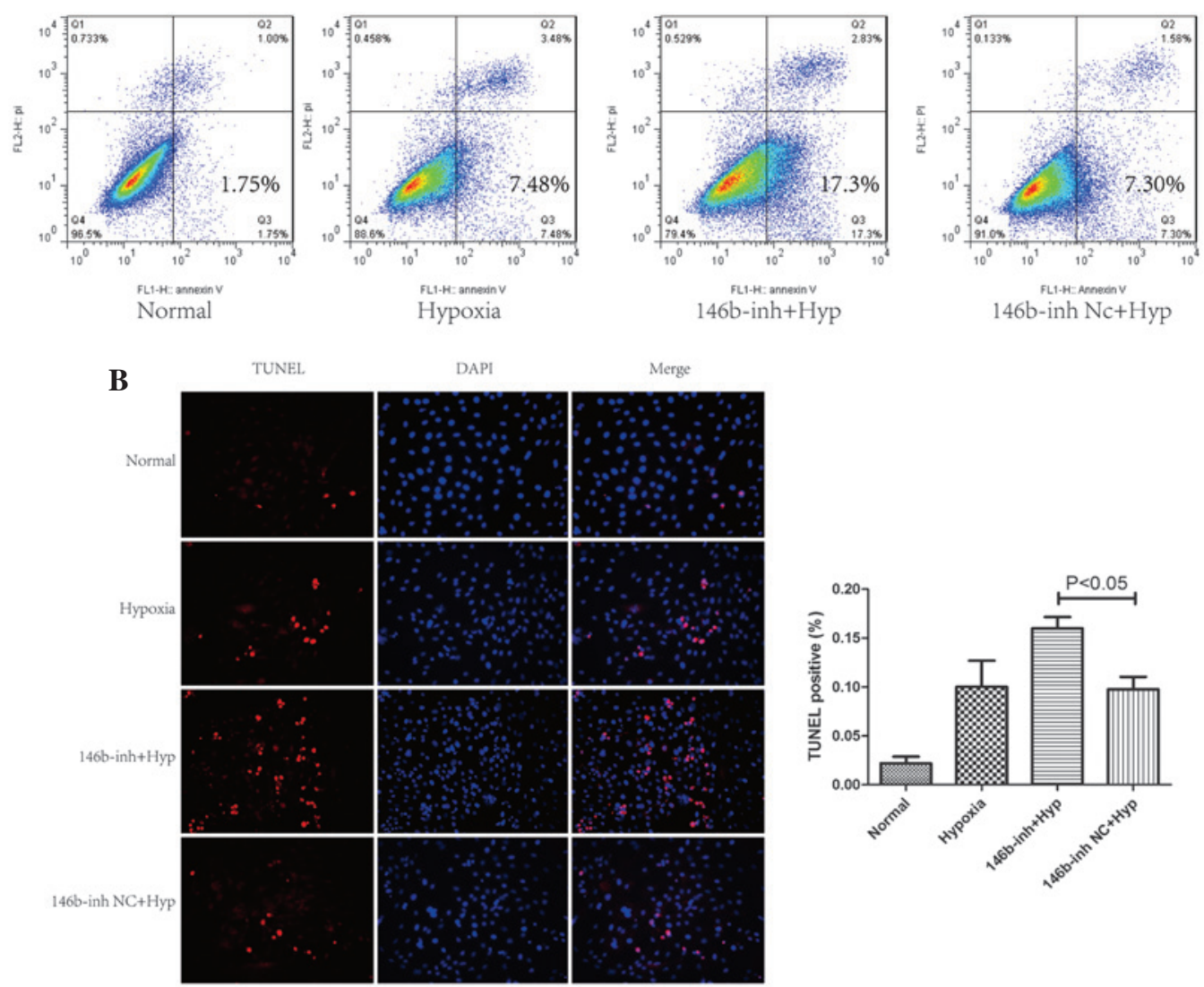

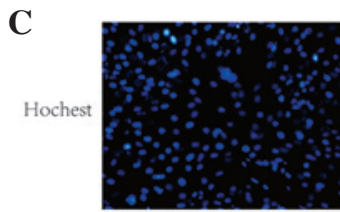

Normal

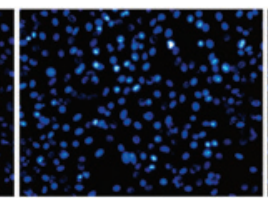

Hypoxia

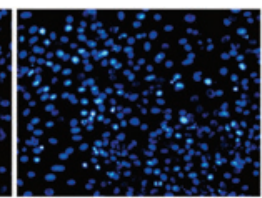

146b-inh+Hyp

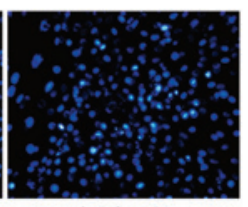

146b-inh $\mathrm{NC}+\mathrm{Hyp}$

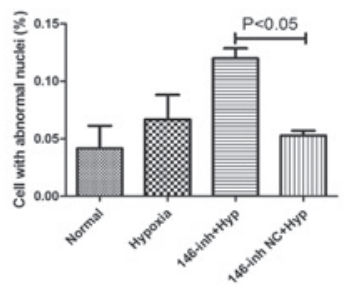

D

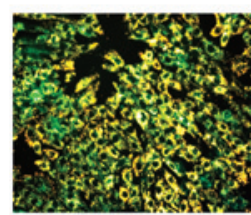

Normal

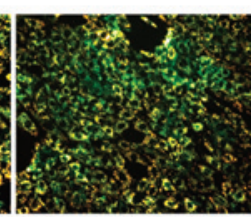

Hypoxia

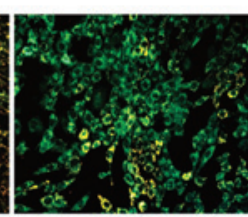

146b-inh+Hyp

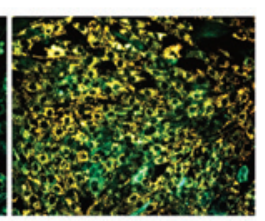

$146 \mathrm{~b}-\mathrm{inh} \mathrm{NC}+\mathrm{Hyp}$

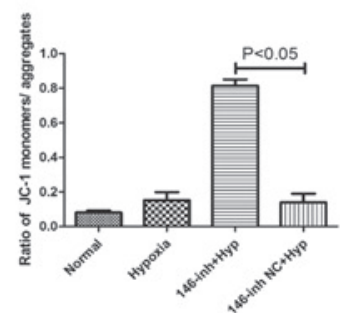

Figure 2. Addition of the miR-146b inhibitor augments the hypoxia-induced apoptosis observed in the H9c2 cells. Following exposure to hypoxia for $72 \mathrm{~h}$, cell apoptosis was detected by (A) annexin V/PI flow cytometric analysis, (B) TUNEL, (C) Hoechst 33258 and (D) JC-1 staining. Each value was counted in five random fields. The data are expressed as the mean \pm standard deviation $(\mathrm{P}<0.05,146 \mathrm{~b}$-inh-treated cells compared with the $\mathrm{NC}$ under hypoxic conditions). NC, normal control; Hyp, hypoxia; PI, propidium iodide; TUNEL, terminal deoxynucleotidyl transferase dUTP nick-end labeling; 146b-inh, miR-146b inhibitor; miR, microRNA.

to $9.75 \pm 1.3 \%$ following the induction of hypoxia $(\mathrm{P}<0.05$, compared with the NC). Transfection with the miR-146b inhibitor further increased the apoptotic rate $(16.00 \pm 1.2 \%$; $\mathrm{P}<0.05)$. Nuclear morphological changes were observed by Hoechst 33258 staining. A larger number of abnormal nuclei were detected in the inhibitor group compared with the $\mathrm{NC}$ group at $72 \mathrm{~h}$ following hypoxia induction (Fig. 2C). JC-1 staining was performed to determine the proportion of cells with a normal $\Delta \Psi_{\mathrm{m}}$. A decreased $\Delta \Psi_{\mathrm{m}}$ was observed following transfection with the miR-146b inhibitor, as revealed by a larger number of cells yielding only a green emission upon excitation (Fig. 2D).

The expression levels of apoptosis-associated proteins were measured by western blotting. The Bcl-2/Bax ratio was decreased in the hypoxia model and was further decreased upon transfection with the miR-146b inhibitor compared with that of the control group (Fig. 3A-D). Transfecting the cells with the miR-146b inhibitor resulted in a significant increase in the levels of cleaved-caspase 3 (Fig. 3A and E) and Bax (Fig. 3A and C), and a decrease in the level of Bcl-2 
A

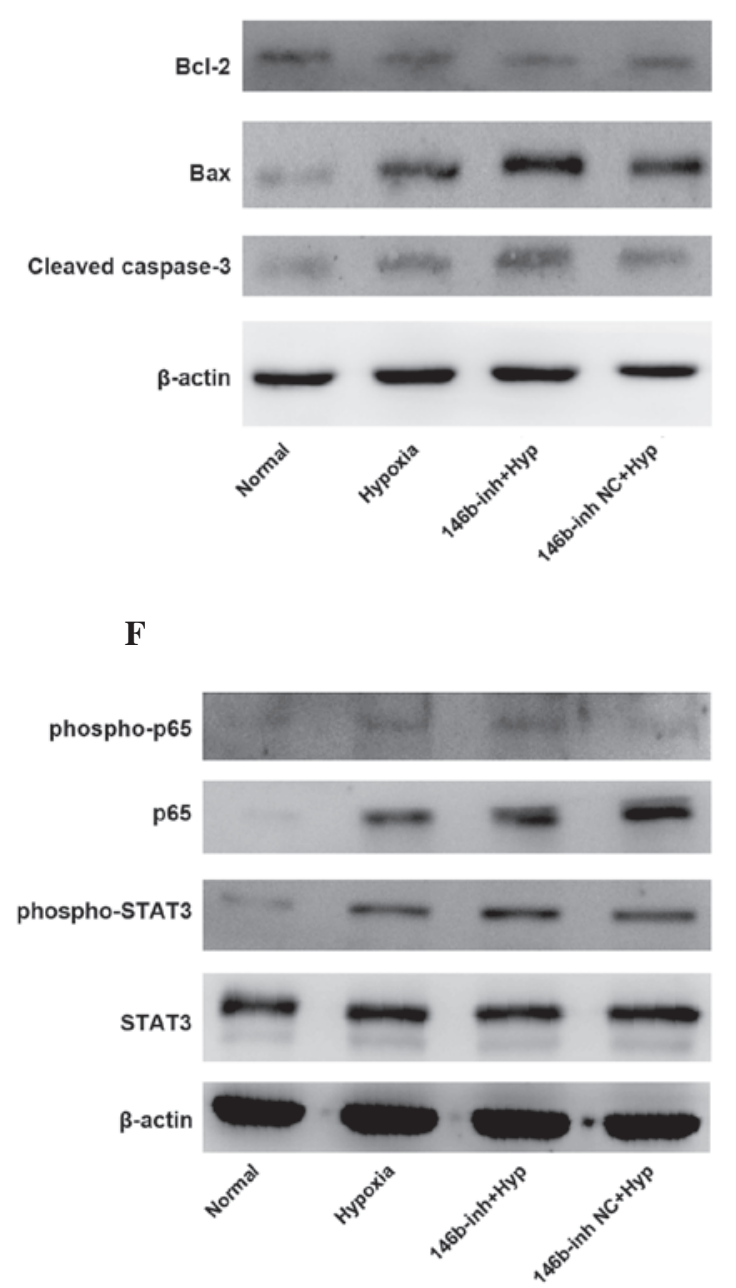

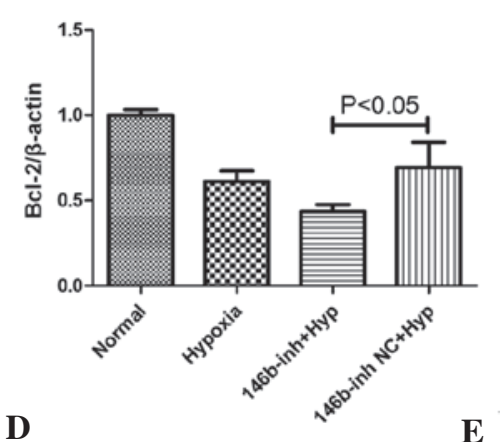

G

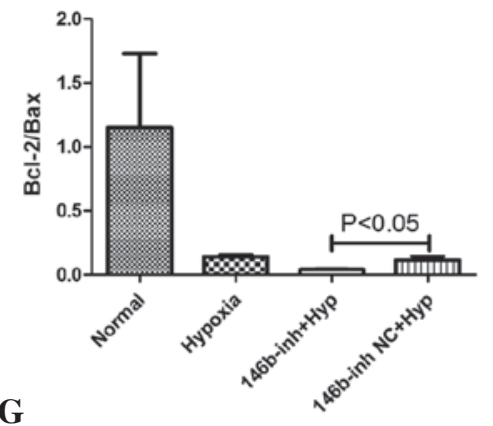

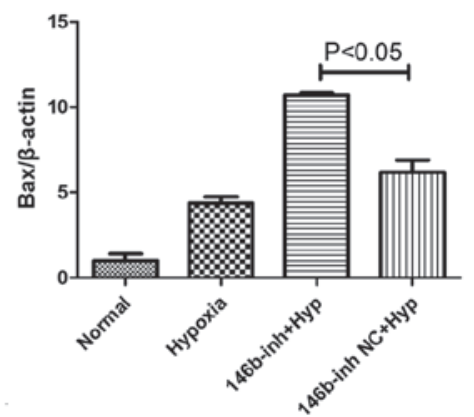
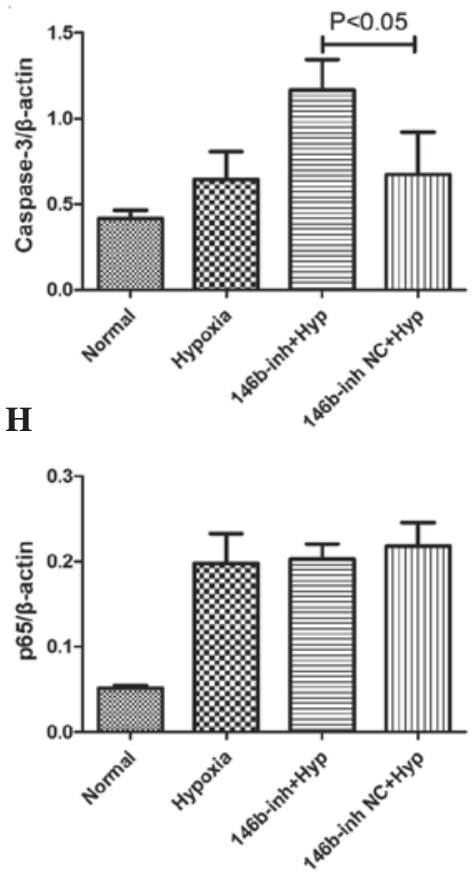

$\mathbf{L}$

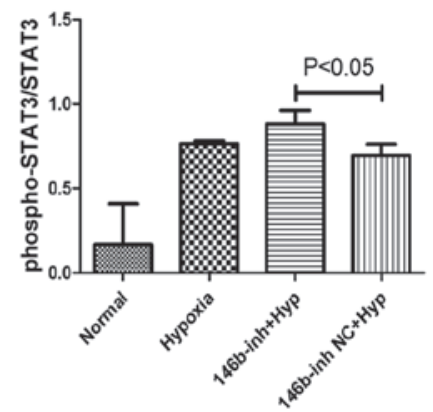

Figure 3. Addition of the miR-146b inhibitor causes changes in the levels of apoptosis-associated proteins and the nuclear factor- $\kappa \mathrm{B} / \mathrm{interleukin}-6 \mathrm{pathway}$. (A) A representative western blot is shown for the Bcl-2, Bax and cleaved-caspase 3 proteins under conditions of normoxia and hypoxia at $72 \mathrm{~h}$ following transfection with the miR-146b inhibitor. The miR-146b inhibitor (B) markedly reduced the expression of Bcl-2, whereas the expression of (C) Bax, (D) the Bcl-2/Bax ratio and (E) cleaved-caspase 3 were increased. (F) Western blot assays were performed for the p65, phospho-p65, STAT3 and phospho-STAT3 proteins under conditions of normoxia and hypoxia at $72 \mathrm{~h}$ following transfection with the miR-146b inhibitor or with NC. The addition of the $146 \mathrm{~b}$-inh $(\mathrm{G})$ significantly increased the expression of phospho-p65, although $(\mathrm{H})$ no significant change in the level of p65 was observed. The levels of (I) phospho-p65/p65 and (J) phospho-STAT3 were significantly increased by the 146b-inh , whereas (K) the level of STAT3 was not significantly altered, although (L) the level of phopsho-STAT3/STAT3 was significantly increased. The values are expressed as the mean \pm standard deviation $(\mathrm{P}<0.05,146 \mathrm{~b}$-inhib -treated cells compared with the NC under hypoxic conditions). NC, normal control; inh, inhibitor; Hyp, hypoxia;phospho, phosphorylated; STAT3, signal transducer and activator of transcription 3; 146b-inhib, miR-146b inhibitor; miR, microRNA.

(Fig. 3A and B), compared with that in the inhibitor-NC group $(\mathrm{P}<0.05)$. These findings indicated that the addition of miR-146b increases the extent of apoptosis in the H9c2 cells under hypoxic conditions.
Inhibition mediated by miR-146b increases the activation of $N F-\kappa B$ and STAT3. miR-146b has been widely recognized to attenuate the activation of the NF- $\kappa B$ signaling pathway. Newly emerging data have revealed a connection between STAT3 and 
A

B
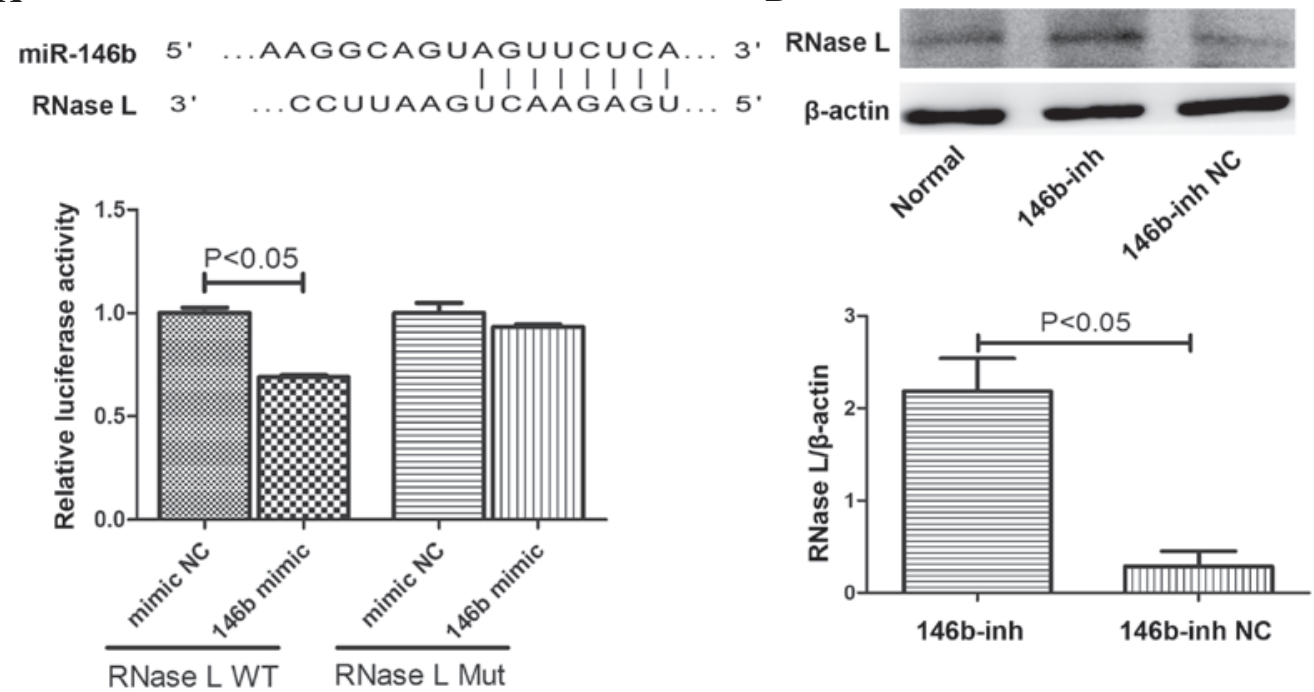

Figure 4. RNase L is a target of miR-146b. (A) The potential binding site of miR-146b in the 3'-UTR of the RNase L mRNA. The luciferase activity was determined at $48 \mathrm{~h}$ following transfection. (B) Western blot analysis revealed that the level of the RNase L protein increased in the 146b-inh group compared with the NC group. The values are expressed as the mean \pm standard deviation $(\mathrm{P}<0.05$, the miR-146b-inh (or mimic)-treated cells compared with the $146 \mathrm{~b}$-inh (or mimic) NC. NC, normal control; Mut, mutation; RNase, ribonuclease; 146b-inh, miR-146b inhibitor; miR, microRNA.

miR-146b (11). The present study revealed that the miR-146b inhibitor failed to cause any significant change in the protein levels of p65 and STAT3 at $72 \mathrm{~h}$ following the induction of hypoxia, although the levels of p-p65 and p-STAT3 were increased compared with the NC group $(\mathrm{P}<0.05$; Fig. 3F-L).

RNase $L$ is a target of $m i R-146 b$. A bioinformatics analysis using TargetScan predicted that RNase $\mathrm{L}$ is a target of miR-146b, as the 3'-UTR of the RNase L mRNA contains a theoretical miR-146b binding site (Fig. 4A). To determine whether miR-146b regulates the expression of RNase $\mathrm{L}$, the 3'-UTR of wild-type or mutated RNase L was cloned into the pmiR-RB-Report vector to generate a luciferase reporter. The miR-146b mimic was subsequently co-transfected with the vector into HEK293T cells and a luciferase activity assay was performed following a further $48 \mathrm{~h}$ incubation. Compared with the control, the miR-146b mimic significantly reduced the normalized luciferase activity $(\mathrm{P}<0.05$; Fig .4B). Furthermore, the protein expression level of RNase $\mathrm{L}$ was increased by the miR-146b inhibitor, suggesting that RNase L is a target of miR-146b by directly binding to its 3'-UTR (Fig. 4B).

\section{Discussion}

Hypoxia causes apoptosis, and a linear association exists between the severity of hypoxic-ischemic cardiomyocyte injury and the number of apoptotic cells (12). In the present study, an increase in the expression of miR-146b was observed in tissue samples of cyanotic CHD and hypoxic H9C2 cells, and the inhibition mediated by miR-146b was revealed to diminish the ability of the H9c2 cells to adapt to chronic hypoxia.

miR-146b differs from miR-146a by having two different nucleotides at its 3 '-end. Although the two miRNAs have similar functions, previous studies revealed that the miR-146 isoforms do exhibit certain specific differences in terms of their expression and biological action $(10,13)$. miR-146a/b repressed the activation of the $\mathrm{NF}-\kappa \mathrm{B}$ pathway by directly targeting IRAK-1, TRAF6 and MyD88 (10). A sustained activation of $\mathrm{NF}-\kappa \mathrm{B}$ was revealed to be proapoptotic in H9c2 cardiomyocytes (14). Therefore, it was hypothesized that inhibition of the NF- $\kappa \mathrm{B}$ pathway caused by upregulated levels of miR-146b may reduce hypoxia-induced cardiomyocyte apoptosis. Furthermore, miR-146a/b targets multiple genes, which are harmful to cardiomyocytes under hypoxic conditions. miR-146a/b targets human antigen R, which suppresses the expression of endothelial nitric oxide synthase (eNOS) (15). Increasing the levels of eNOS inhibited hypoxia-induced apoptosis, therefore, leading to a lower rate of apoptosis (16). miR-146a/b targets the endothelial growth factor receptor (17), which may increase hypoxia-induced cardiomyocyte injury. miR-146a was previously identified as having a protective role in cardiac I/R injury. It was demonstrated that, although the expression of miR-146a does not notably increase following I/R injury, the overexpression of miR-146a may significantly reduce the I/R injury, which is partly caused by the attenuation of both the activation of NF- $\mathrm{KB}$ and inflammatory cytokine production by suppressing IRAK1 and TRAF6 (18). miR-146 is a therapeutic target and biomarker for peripartum cardiomyopathy (19). In the present study, miR-146b was revealed to protect cardiomyocytes from hypoxia-induced apoptosis.

A previous report revealed that miR-146b exerts a critical role in the feedback communication between $\mathrm{NF}-\kappa \mathrm{B}$ and the interleukin-6 (IL-6) signaling axis (20). miR-146b acts as a feedback regulator of this pathway, leading to the inhibition of $\mathrm{NF}-\kappa \mathrm{B}$ (11). A previous study in our laboratory reported that the expression levels of p65, p-p65 and p-STAT3 were increased following the onset of chronic hypoxia in cardiomyocytes (21), findings which are corroborated by the results in the present study. STAT3 exerts a cardioprotective role in the ischemic heart (22), and miR-146b is a target gene of STAT3 (11). The upregulation of miR-146b may finely adjust the activation of NF- $\kappa \mathrm{B}$ in the IL- 6 signaling axis, in addition 
to other target genes, thereby acting as a protective regulator in chronic hypoxia.

RNase L is a member of the 2-5A system, and it belongs to the Mx family. RNase L may induce apoptosis in a caspase-dependent manner. Furthermore, RNase L may change the $\Delta \Psi_{\mathrm{m}}$ and elicit the generation of reactive oxygen species (23). RNase L-deficient cells are highly resistant to apoptosis, which is partly mediated through the c-Jun $\mathrm{N}$-terminal kinase/c-jun pathway (24). RNase L may also affect the NF- $\kappa \mathrm{B}$ signaling pathways by indirectly affecting the I $\kappa$ B kinases- $\alpha$ and $-\varepsilon(25)$. As revealed in the Tissue-specific Gene Expression and Regulation database, the mRNA expression levels of RNase L are relatively high in heart tissue, suggesting that it may fulfil an important role in pathophysiological processes of the heart.

The primary role of the $\mathrm{Bcl}-2$ protein family is to regulate cell apoptosis. In this family, Bax is proapoptotic, whereas Bcl-2 is antiapoptotic (26). The ratio of Bcl-2/Bax acts as an indicator of the mortality or survival of the cells, following an apoptotic stimulus (27). The caspase protein family are essential mediators of apoptosis. Among the family members, caspase 3 is the key protease, which is involved in the final execution phase of apoptosis (28). It was previously reported that the Bcl-2 family of proteins and caspase- 3 are involved in the RNase L-mediated apoptotic pathway. The activation of RNase L led to the release of cytochrome $c$ from the mitochondria, and adding a caspase 3 inhibitor or overexpressing $\mathrm{Bcl}-2$ suppressed the RNase L-mediated apoptosis (29). The present study revealed that the inhibition mediated by $\mathrm{miR}-146 \mathrm{~b}$ regulated these apoptosis-associated proteins, causing an increased expression of cleaved-caspase 3 and Bax and a decreased expression of Bcl-2 and Bcl-2/Bax, suggesting that miR-146b may exerts its influence by targeting RNase L.

Several questions remain unanswered by the present study. Only the downregulation, and not the upregulation, of miR-146b was investigated in this study. The expression levels of routine miR-146 targets, including IRAK1 and TRAF6, were not investigated, since their roles under hypoxic conditions are well established.

Taken together, the present study indicated that the inhibition of miR-146b increased hypoxia-induced apoptosis, which may be partly caused by its target, RNase L. miR-146b has a protective role in cardiac chronic hypoxia.

\section{Acknowledgements}

This study was supported by the National Science Foundation of China (no. 81270228) The authors would like to thank Miss Fu-Qing Tang for her technical assistance. (Department of Cardiovascular Surgery, PLA, Xinqiao Hospital, Third Military Medical University)

\section{References}

1. Jian Z, Li JB, Ma RY, Chen L, Zhong QJ, Wang XF, Wang W, Hong $\mathrm{Y}$ and $\mathrm{Xiao} \mathrm{YB}$ : Increase of macrophage migration inhibitory factor (MIF) expression in cardiomyocytes during chronic hypoxia. Clin Chim Acta 405: 132-138, 2009.

2. Waskova-Arnostova P, Kasparova D, Elsnicova B, Novotny J, Neckar J, Kolar F and Zurmanova J: Chronic hypoxia enhances expression and activity of mitochondrial creatine kinase and hexokinase in the rat ventricular myocardium. Cell Physiol Biochem: 33: 310-320, 2014.
3. Hausser $\mathbf{J}$ and Zavolan $\mathrm{M}$ : Identification and consequences of miRNA-target interactions - beyond repression of gene expression. Nat Rev Genet 15: 599-612, 2014.

4. Condorelli G, Latronico MV and Cavarretta E: microRNAs in cardiovascular diseases: Current knowledge and the road ahead. J Am Coll Cardiol 63: 2177-2187, 2014.

5. He S, Liu P, Jian Z, Li J, Zhu Y, Feng Z and Xiao Y: miR-138 protects cardiomyocytes from hypoxia-induced apoptosis via MLK3/JNK/c-jun pathway. Biochem Biophys Res Commun 441: 763-769, 2013.

6. Zhang J, Chang JJ, Xu F, Ma XJ, Wu Y, Li WC, Wang HJ, Huang GY and Ma D: MicroRNA deregulation in right ventricular outflow tract myocardium in nonsyndromic tetralogy of fallot. Can J Cardiol 29: 1695-1703, 2013.

7. Chan SY, Zhang YY, Hemann C, Mahoney CE, Zweier JL and Loscalzo J: MicroRNA-210 controls mitochondrial metabolism during hypoxia by repressing the iron-sulfur cluster assembly proteins ISCU1/2. Cell Metab 10: 273-284, 2009.

8. Wang D, Liu YL, Lü XD, Ling F, Liu AJ, Du J and Han L: Lung microRNA profile in chronic cyanotic piglets with decreased pulmonary blood flow. Chin Med J (Engl) 126: 2260-2264, 2013.

9. Taganov KD, Boldin MP, Chang KJ and Baltimore D: NF-kappaB-dependent induction of microRNA miR-146, an inhibitor targeted to signaling proteins of innate immune responses. Proc Natl Acad Sci USA 103: 12481-12486, 2006.

10. Curtale G, Mirolo M, Renzi TA, Rossato M, Bazzoni F and Locati M: Negative regulation of Toll-like receptor 4 signaling by IL-10-dependent microRNA-146b. Proc Natl Acad Sci USA 110: 11499-11504, 2013

11. Xiang M, Birkbak NJ, Vafaizadeh V, Walker SR, Yeh JE, Liu S, Kroll Y, Boldin M, Taganov K, Groner B, et al: STAT3 induction of miR-146b forms a feedback loop to inhibit the NF-kappaB to IL-6 signaling axis and STAT3-driven cancer phenotypes. Sci Signal 7: ra11, 2014

12. Mehmet $\mathrm{H}$ and Edwards AD: Hypoxia, ischaemia, and apoptosis. Arch Dis Child Fetal Neonatal Ed 75: F73-F75, 1996.

13. Perry MM, Williams AE, Tsitsiou E, Larner-Svensson HM and Lindsay MA: Divergent intracellular pathways regulate interleukin-1beta-induced miR-146a and miR-146b expression and chemokine release in human alveolar epithelial cells. FEBS Lett 583: 3349-3355, 2009.

14. Hamid T, Gu Y, Ortines RV, Bhattacharya C, Wang G, Xuan YT and Prabhu SD: Divergent tumor necrosis factor receptor-related remodeling responses in heart failure: Role of nuclear factor-kappaB and inflammatory activation. Circulation 119: 1386-1397, 2009

15. Cheng HS, Sivachandran N, Lau A, Boudreau E, Zhao JL, Baltimore D, Delgado-Olguin P, Cybulsky MI and Fish JE: MicroRNA-146 represses endothelial activation by inhibiting pro-inflammatory pathways. EMBO Mol Med 5: 949-966, 2013.

16. Wang H, Zuo X, Wang Q, Boudreau E, Zhao JL, Baltimore D, Delgado-Olguin P, Cybulsky MI and Fish JE: Nicorandil inhibits hypoxia-induced apoptosis in human pulmonary artery endothelial cells through activation of mitoKATP and regulation of eNOS and the NF-kappaB pathway. Int J Mol Med 32: 187-194, 2013.

17. Hurst DR, Edmonds MD, Scott GK, Benz CC, Vaidya KS and Welch DR: Breast cancer metastasis suppressor 1 up-regulates miR-146, which suppresses breast cancer metastasis. Cancer Res 69: 1279-1283, 2009.

18. Wang X, Ha T, Liu L, Zou J, Zhang X, Kalbfleisch J, Gao X, Williams D and Li C: Increased expression of microRNA-146a decreases myocardial ischaemia/reperfusion injury. Cardiovasc Res 97: 432-442, 2013.

19. Halkein J, Tabruyn SP, Ricke-Hoch M, Haghikia A, Nguyen NQ, Scherr M, Castermans K, Malvaux L, Lambert V, Thiry M, et al: MicroRNA-146a is a therapeutic target and biomarker for peripartum cardiomyopathy. J Clin Invest 123: 2143-2154, 2013.

20. Walker SR, Xiang M and Frank DA: STAT3 Activity and Function in Cancer: Modulation by STAT5 and miR-146b. Cancers (Basel) 6: 958-968, 2014.

21. Gu Q, Kong Y, Yu ZB, Bai L and Xiao YB: Hypoxia-induced SOCS3 is limiting STAT3 phosphorylation and NF- $\mathrm{KB}$ activation in congenital heart disease. Biochimie 93: 909-920, 2011.

22. Hilfiker-Kleiner D, Hilfiker A, Fuchs M, Kaminski K, Schaefer A, Schieffer B, Hillmer A, Schmiedl A, Ding Z, Podewski E, et al: Signal transducer and activator of transcription 3 is required for myocardial capillary growth, control of interstitial matrix deposition, and heart protection from ischemic injury. Circ Res 95: $187-195,2004$. 
23. Domingo-Gil E and Esteban M: Role of mitochondria in apoptosis induced by the 2-5A system and mechanisms involved. Apoptosis 11: 725-738, 2006

24. Malathi K, Paranjape JM, Ganapathi R and Silverman RH: HPC1/RNASEL mediates apoptosis of prostate cancer cells treated with 2',5'-oligoadenylates, topoisomerase I inhibitors, and tumor necrosis factor-related apoptosis-inducing ligand. Cancer Res 64: 9144-9151, 2004.

25. Chakrabarti A, Jha BK and Silverman RH: New insights into the role of RNase L in innate immunity. J Interferon Cytokine Res 31: 49-57, 211.

26. Siddiqui WA, Ahad A and Ahsan H: The mystery of BCL2 family: Bcl-2 proteins and apoptosis: an update. Arch Toxicol 89: 289-317, 2015.
27. Korsmeyer SJ, Shutter JR, Veis DJ, Merry DE and Oltvai ZN: Bcl-2/Bax: A rheostat that regulates an anti-oxidant pathway and cell death. Semin Cancer Biol 4: 327-332, 1993.

28. Shalini S, Dorstyn L, Dawar S and Kumar S: Old, new and emerging functions of caspases. Cell Death Differ 22: 526-539, 2015.

29. Li G, Xiang Y, Sabapathy K and Silverman RH: An apoptotic signaling pathway in the interferon antiviral response mediated by RNase L and c-Jun NH2-terminal kinase. J Biol Chem 279: $1123-1131,2004$ 